\title{
The anti-vaccination movement: when does a belief become delusional?
}

\author{
Adam J. Fusick ${ }^{1}$ (D) - Steven Gunther ${ }^{1} \cdot$ Gregory Sullivan $^{1}$ \\ Received: 17 December 2019 / Accepted: 21 February 2020 / Published online: 11 March 2020 \\ (C) This is a U.S. government work and not under copyright protection in the U.S.; foreign copyright protection may apply 2020
}

'Delusion' is a term that can be applied colloquially to any farfetched or often generally unaccepted idea. Clinicians, by contrast, use the term to clearly delineate a specific form of thought content, based on particular criteria. The Diagnostic Statistic Manual of Mental Health Disorders 5th edition (DSM-V) defines delusion as: fixed beliefs that are not amenable to change in light of conflicting evidence (American Psychiatric Association 2013). Further, this pattern of thought is not in accordance with the individual's culture or sub-culture. This definition has difficulties with many strange beliefs, from the catastrophic thoughts of patients with Obsessive Compulsive Disorder to conspiracy theorists postulating the whereabouts of extraterrestrials. Alternatively, the classification of an 'overvalued idea' was first described by Wernicke as an abnormal belief that is neither delusional nor obsessional in nature, but which is preoccupying to the extent of dominating the sufferer's life (Mckenna 1984). The DSM-V, while vague in its definition, also offers the view that an overvalued idea is an unreasonable and sustained belief that is maintained with less than delusional intensity, i.e., the person is able to acknowledge the possibility that the belief may not be true (American Psychiatric Association 2013).

Using these definitions, it is clear that not every belief would neatly fit into one of these categories. For instance, a persecutory belief of being monitored by the police could be common to both an individual with schizophrenia as well as someone recently engaged in criminal activity. Obviously, knowledge of the individual's reasoning, detail of thought, and conviction would be necessary in order to differentiate. As a noteworthy example, currently there is significant

Adam J. Fusick

adam.fusick@va.gov

1 Mental Health and Behavioral Sciences Service, James A. Haley Veterans Hospital, 13000 Bruce B. Downs Boulevard \#116A, Tampa, FL 33612, USA suspicion and opposition regarding vaccinations. Despite substantial evidence that the benefits of vaccination greatly outweigh the risk, there still exists an anti-vaccination movement. 'Anti-Vaxx' beliefs have been highlighted, with notable examples of the Rockland, New York suburb needing to declare a state of emergency, and the United States facing a growing rate of measles, despite its eradication in 2000 (Reiss 2019; Sun 2019).

In evaluating the thought behind the Anti-Vaxx movement, it is clear that these ideas persist despite evidence to the contrary, and there is a sub-culture of individuals who share this belief. The research that help fuel the modern version of this movement by Andrew Wakefield has been publicly discredited, and the egregious flaws in his research ultimately resulted in his removal from the UK Medical Registry (Hussain et al. 2018). Despite this, Anti-Vaxxers hold steadfast in their beliefs, now resulting in multiple outbreaks of measles that have contributed to bodily harm and even death. This would clearly fall outside the boundaries of Wernicke's definition of "...dominating the sufferer's life", as others are now being affected, as well as outside the DSM definition of “...less than delusional intensity".

As some beliefs have always remained difficult to classify, there is little guidance in how to best identify this spectrum of false ideas. There does exist a body of literature debating the potential range of false beliefs, which introduces the term 'delusion-like' ideas. Chaudhury and Kiran nicely summarize this historical approach to this classification issue, and provide the proposition that "an intellectual sleight of hand is often in operation in the distinction of overvalued and delusion-like. If we intend to make a 'psychotic' diagnosis, then the belief is delusion-like; if we intend to make a 'non-psychotic' diagnosis, then the belief is overvalued" (Chaudhury and Kiran 2009).

This perceived ambiguity is perhaps the reason that a more appropriate classification system does not exist. This ambiguity is displayed by varying definitions of 'overvalued idea'. Wernicke's original definition, for example, came with a 
caveat that the idea is shared by others (Mckenna 1984). This understanding directly contradicts the DSM-V definition, where the idea must not be shared by others in order meet diagnostic criteria (American Psychiatric Association 2013). Overall, an unclear array of criteria probably only serves to further cloud the diagnostic picture, and highlights the disparity that exists for clinicians.

Appropriate identification of false ideas is paramount for clinicians. The Anti-Vaxx movement has shown both unwavering conviction in their beliefs as well as an extensive and dangerous series of consequences from their associated actions. If unintended harm is now coming to others because of an unwavering false belief, despite overwhelming evidence to the contrary, is there now a need to advocate for a more complete classification system?

\section{Compliance with ethical standards}

The authors declare that they have no conflict of interest. This article does not contain any studies with human participants or animals performed by any of the authors, and no informed content was needed in the creation of this manuscript.

\section{References}

American Psychiatric Association (2013) Diagnostic and statistical manual of mental disorders: Dsm-5. American Psychiatric Association, Arlington, VA

Chaudhury S, Kiran C (2009) Understanding delusions. Ind Psychiatry J 18(1):3. https://doi.org/10.4103/0972-6748.57851

Hussain A, Ali S, Ahmed M, Hussain S (2018) The anti-vaccination movement: a regression in modern medicine. Cureus 10(7):e2919. https://doi.org/10.7759/cureus.2919

Mckenna P (1984) Disorders with overvalued ideas. Br J Psychiatry 145(6):579-585. https://doi.org/10.1192/bjp.145.6.579

Reiss, D (2019). New York county's move on vaccinations was bold, necessary and perfectly legal. CNN. Retrieved from https://www. cnn.com/2019/03/30/opinions/vaccinations-new-york-ban-reiss/ index.html. Accessed 01 Oct 2019

Sun, LH (2019). U.S. measles cases surge to second-highest level in nearly two decades. Washington Post. Retrieved from https://www. washingtonpost.com/health/2019/04/01/us-measles-cases-surgesecond-highest-level-nearly-two-decades/?noredirect=on. Accessed 01 Oct 2019

Publisher's note Springer Nature remains neutral with regard to jurisdictional claims in published maps and institutional affiliations. 